\title{
Q Wave and Non-Q Wave Myocarditis with Special Reference to Clinical Significance
}

\author{
Hiroshi NAKashima, MD, Tomoyuki KaTAYAMA, MD, \\ Masahiko IsHIZAKI, MD, Masayoshi TAKENO, MD, \\ Yukiharu HONDA, MD, \\ and Katsusuke Yano, ${ }^{\prime} \mathrm{MD}$
}

\section{SUMMARY}

This study was designed to evaluate the differences in clinical findings between patients with and without $Q$ waves in acute myocarditis. Among a total of 24 patients, eleven patients had $Q$ waves and thirteen did not. Echocardiographic findings, in-hospital complications and follow-up results were compared between the two groups. In the acute stage, the $Q$ wave group showed significantly higher creatine kinase $(\mathrm{CK})$ values and a more impaired left ventricular ejection fraction than the non- $Q$ wave group $(40 \pm 11 \%$ vs $57 \pm 10 \%, p<0.001)$. Transient left ventricular hypertrophy was also prominent in the $Q$ wave group. The incidence of cardiogenic shock $(55 \%)$ and conduction disturbances $(64 \%)$ were higher in the $Q$ wave group than in the non-Q wave group ( $0 \%$ and $15 \%$, respectively). In-hospital mortality rate was $27 \%$ in the $Q$ wave group and $8 \%$ in the non- $Q$ wave group, respectively. Since rapid improvement occurred in survivors with $Q$ waves, long-term prognosis was favorable for the two groups. In conclusion, $Q$ waves might indicate a more severe course in early illness. (Jpn Heart J 1998; 39: 763-774)

Key words: Myocarditis, Abnormal $Q$ waves, Creatine kinase, Echocardiographic findings, In-hospital complications

$\mathrm{E}$ LECTROCARDIOGRAPHIC findings in acute myocarditis show widespread ST deviations, conduction disturbances, and abnormal $Q$ waves which sometimes imitate acute myocardial infarction. ${ }^{1-4)}$ The incidence of abnormal $Q$ waves ranges widely from 14 to $63 \%$ in myocarditis. ${ }^{1,5-7)}$ A transient appearance of $Q$ waves during an acute illness ${ }^{1,7)}$ may indicate gross, but reversible myocardial damage. To our knowledge, previous studies give little attention to the differences in clinical characteristics between patients with $Q$ wave myocarditis and non- $Q$ wave myocarditis. This study was designed on the hy-

From the Department of Cardiology, Nagasaki Citizens Hospital, Nagasaki and 'Third Department of Internal Medicine, Nagasaki University School of Medicine, Nagasaki, Japan.

Address for correspondence: Hiroshi Nakashima, MD, Department of Cardiology, Nagasaki Citizens Hospital, 6-39 Shinchi-machi, Nagasaki 850, Japan.

Received for publication August 24, 1998.

Revised and accepted November 11, 1998. 
pothesis that $Q$ wave myocarditis might show more extensive myocardial damage and severe left ventricular dysfunction compared to non- $Q$ wave myocarditis. Consequently, a poorer clinical outcome might occur in patients with $Q$ wave myocarditis.

\section{Materials and Methods}

Study subjects and diagnosis: Forty two patients with clinically suspected acute myocarditis were admitted to our hospital between January 1990 and December 1996. In the present study, a diagnosis of acute myocarditis was required to meet the following criteria: 1) new cardiac abnormalities developed following symptoms of an acute viral infection; 2) evolutional electrocardiographic changes; 3) impaired left ventricular function on the echocardiogram; 4) absence of persistent left bundle branch block which obscured recognition of the abnormal $Q$ waves; 5) normal coronary arteriograms; 6) biopsy-proven myocarditis according to the Dallas criteria ${ }^{8)}$ or a positive pyrophosphate scan; 7) absence of valvular heart disease and other systemic diseases such as diabetes mellitus, collagen disease, or sarcoidosis.

Consequently, 24 patients met the above criteria and were included in this study. There were 17 men and 7 women with a mean age of $51 \pm 16$ years. Seventeen of 24 patients showed positive biopsy findings and 7 patients revealed a positive pyrophosphate scan. The diagnoses were further supported by fluctuated virus antibody titer. Neutralizing antibody titer to Coxsackie B and echo viruses were examined in 21 patients. Eleven patients showed greater than fourfold fluctuations in antibody titer.

Abnormal $Q$ wave was defined as $>40 \mathrm{msec}$. Based on this criteria, study patients were classified into either the $Q$ wave group $(n=11)$ or the non- $Q$ wave group ( $n=13$ ). CK had been measured at least twice daily for 5 days after admission. The maximum level and the time course of $\mathrm{CK}$ values were evaluated for the two groups.

Echocardiography: All patients underwent echocardiographic examination on admission and at 3 or 4 weeks following admission (convalescent stage) except two patients in the $Q$ wave group who died within one week. Left ventricular end-diastolic and end-systolic diameter, and end-diastolic wall thickness were measured on the M-mode echocardiogram according to the recommendations of the American Society of Echocardiography. ${ }^{9}$ Left ventricular volumes were calculated from these data using the method of Teichholtz et al. ${ }^{10)}$ and left ventricular ejection fraction was estimated. Left ventricular wall thickness was expressed by the sum of end-diastolic interventricular septum and left ventricular posterior wall thickness. To assess segmental asynergy of left ventricular systolic perfor- 
mance, the two-dimensional echocardiographic examination was used.

Intravenous methylprednisolone ( $1,000 \mathrm{mg} /$ day) was given for 3 days to five patients with $Q$ waves myocarditis because of refractory heart failure as well as advanced conduction disturbances. Oral prednisolone was then initiated at 30 $\mathrm{mg} /$ day in divided oral doses and reduced over the next 6 weeks to withdrawal of the drug in 3 patients. A comparison of left ventricular function before and at 4 days after treatment was made.

Definitions of hemodynamics and conduction disturbances: Pulmonary edema was defined by characteristic shadows on the chest roentgenogram. ${ }^{117}$ Cardiogenic shock was defined as 1) arterial systolic blood pressure lower than 90 $\mathrm{mm} \mathrm{Hg}$ without inotropic or intraaortic balloon pump support; 2) absence of hypovolemia; 3) peripheral circulatory collapse: clammy skin, urine flow less than $30 \mathrm{ml} / \mathrm{h}$. Left and right bundle branch blocks were diagnosed by the definitions of the Federation for Cardiology Task Force. ${ }^{12)}$ Advanced atrioventricular block was diagnosed when 3:1 or more atrioventricular block was present.

Follow-up: Follow-up data of twenty patients who survived an acute illness were obtained from medical records (15 patients) or telephone contact (5 patients). Cardiac death, NYHA class, and readmission because of heart failure were compared between the two groups.

Statistics: The results are expressed as mean $\pm \mathrm{SD}$. Continuous variables were compared by using Student's $t$ test for paired and unpaired data as appropriate. Simple regression analysis was performed to examine the relation of left ventricular ejection fraction and left ventricular wall thickness or the number of leads with $\mathrm{Q}$ waves. Discrete variables were analyzed by $\chi^{2}$ test. Statistical significance was defined at $p<0.05$.

\section{Results}

Patient characteristics: The baseline clinical characteristics are shown in Table I. All $Q$ waves in the $Q$ wave group appeared within 3 days after admission. Subsequently, $Q$ waves in survivors disappeared over the 4 weeks, except in one patient in whom $Q$ waves were noted 52 days later. There was no significant difference between the two groups in age and gender. Patients with $Q$ waves exhibited a higher incidence of NYHA class III or IV on admission (100\% vs $46 \% ; p=0.004$ ). Systolic blood pressure was significantly lower in the $Q$ wave group than in the non- $Q$ wave group. Heart rate and $C$-reactive protein were comparable in the two groups. ST elevation was more frequent in the $\mathbf{Q}$ wave group than in the non-Q wave group $(82 \%$ vs $0 \%, p<0.001)$. All patients in the non- $Q$ wave group showed ST depression and/or negative $T$ waves. The $Q$ wave group showed significantly higher maximum CK values. Patients with $Q$ 
Table I. Patient Characteristics

\begin{tabular}{lccc}
\hline & $\begin{array}{c}\text { Q wave group } \\
(n=11)\end{array}$ & $\begin{array}{c}\text { Non-Q wave group } \\
(n=13)\end{array}$ & $p$ \\
\hline Age $(y)$ & $54 \pm 12$ & $48 \pm 18$ & 0.388 \\
Male & $9(82)$ & $8(62)$ & 0.089 \\
NYHA $\geqq$ Class III & $11(100)$ & $8(62)$ & 0.004 \\
Systolic BP, mm Hg & $106 \pm 24$ & $133 \pm 20$ & 0.004 \\
Heart rate, bpm & $92 \pm 32$ & $73 \pm 16$ & 0.093 \\
Maximum CK, IU/l & $680 \pm 559$ & $256 \pm 284$ & 0.025 \\
CRP, mg/d $l$ & $5.2 \pm 2.8$ & $4.6 \pm 7.3$ & 0.770 \\
Inotropic agents & $8(73)$ & $1(8)$ & 0.001 \\
Corticosteroids & $5(45)$ & $0(0)$ & 0.036 \\
Temporary pacing & $6(55)$ & $1(8)$ & 0.016 \\
Intraaoric balloon pump & $2(18)$ & $0(0)$ & 0.122 \\
Mortality rate & $3(27)$ & $1(8)$ & 0.200 \\
\hline
\end{tabular}

( ) : \% $\mathrm{BP}=$ blood pressure; $\mathbf{C K}=$ creatine kinase; $\mathrm{CRP}=\mathrm{C}$-reactive protein.
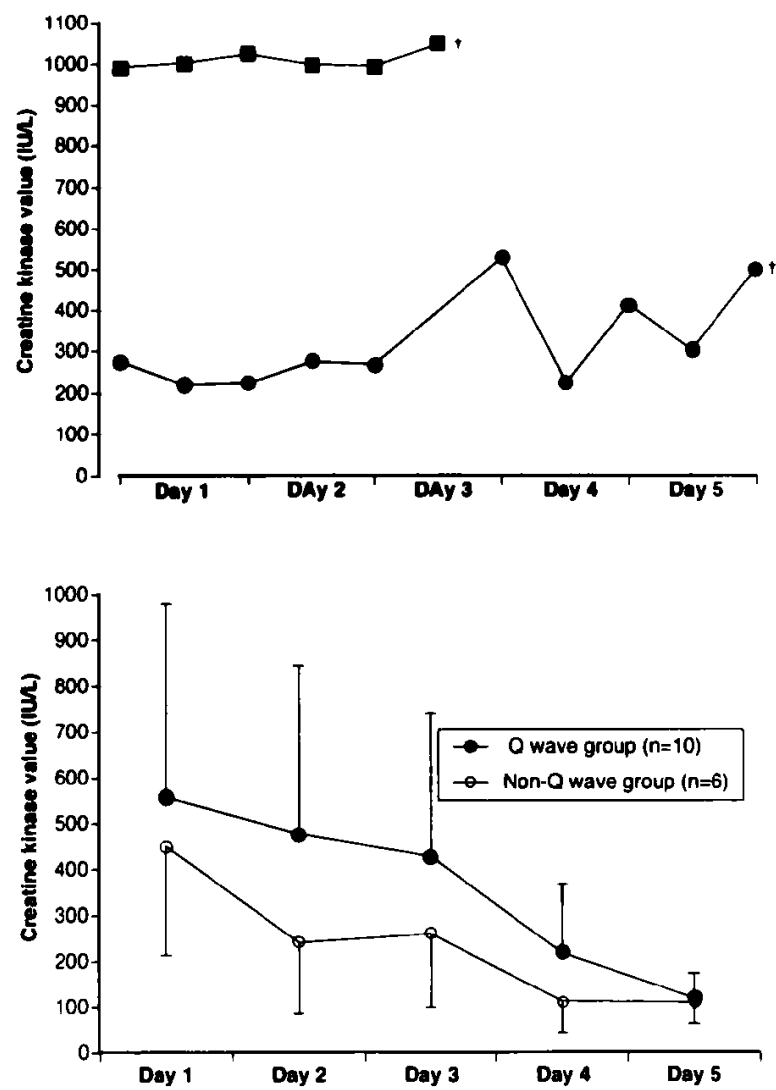

Figure 1. Upper panel: Time course of $\mathrm{CK}$ values in two representive patients with $Q$ wave myocarditis. Lower panel: A comparison of time courses of CK values of the two groups. Closed circle $=Q$ wave group; Open circle $=$ non- $Q$ wave group. 
wave myocarditis required significantly more intensive care such as inotropic agents, corticosteroids, and temporary pacing compared with non- $Q$ wave myocarditis. In-hospital mortality rate was higher in the $Q$ wave group than in the non- $Q$ wave group, however, this difference did not reach statistical significance.

Time course of CK values: Two representative time courses of CK values in the $Q$ wave group are shown in Figure 1; upper panel. These two patients died within 7 days. One patient had persistently high $\mathrm{CK}$ values around 1,000 IU/l for three days. The other patient showed three-fold re-elevations of CK values and they did not return to normal values for 5 days. In severe myocarditis patients, CK values did not show a peak point and high CK values had persisted for a relatively long period associated with later re-elevation. These features were quite different from those of acute myocardial infarction. A comparison of the two groups are shown in Figure 1; lower panel. Ten of 11 patients (91\%) with $Q$ wave myocarditis and 6 of 13 patients $(46 \%)$ with non-Q wave myocarditis exhibited abnormal CK values over $220 \mathrm{IU} / l(p=0.020)$. At each point for 4 days, the $C K$ values were higher in patients with $Q$ wave myocarditis than in those with non- $Q$ wave myocarditis, however, this difference did not reach statistical significance. Time to normal CK values also did not differ in the two groups $(75 \pm 11 \mathrm{~h}$ vs $50 \pm 27 \mathrm{~h}, p=0.208)$.

Echocardiographic findings and effects of corticosteroid treatment on left
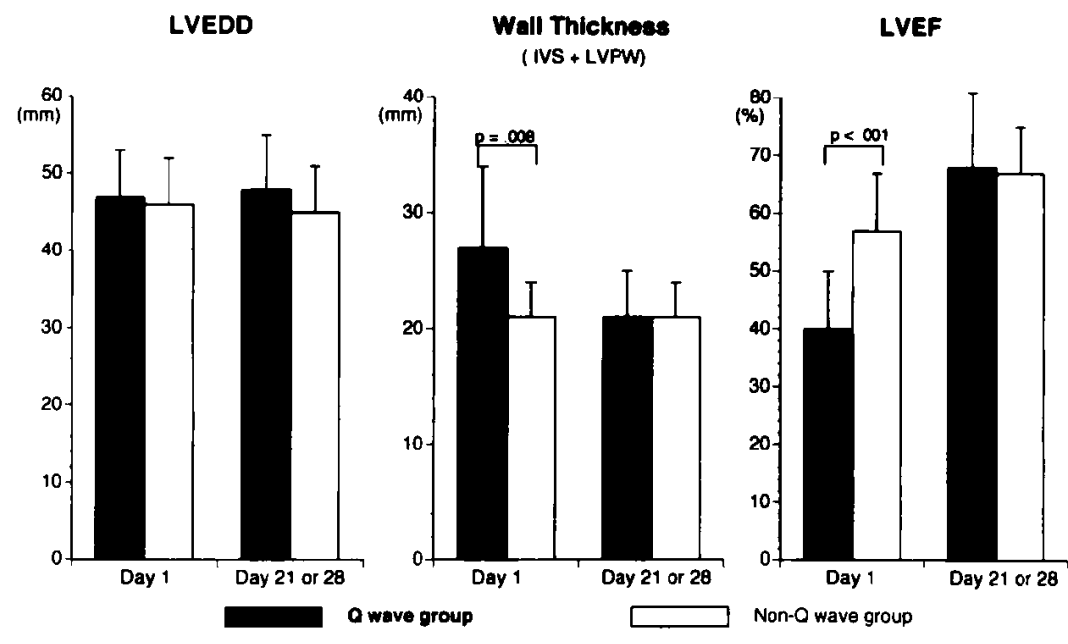

Figure 2. Comparison of echocardiographic findings of the two groups. The $Q$ wave group shows a significantly decreased left ventricular ejection fraction and a prominent myocardial thickening in the acute stage. IVS = interventricular septum; LVPW = left ventricular posterior wall; Closed column = $Q$ wave group; Open column $=$ non- $Q$ wave group. 
ventricular function: Left ventricular function estimated by echocardiogram is shown in Figure 2. On admission, the left ventricular end-diastolic diameters were comparable in the two groups. The $\mathrm{Q}$ wave group showed a significantly higher wall thickness $(p=0.008)$ and a depressed left ventricular ejection fraction ( $p=0.001$ ) compared with the non- $Q$ wave group. The relation between wall thickness and left ventricular ejection fraction showed a significant inverse correlation $(r=0.458, p=0.028)$. Segmental left ventricular systolic dysfunction could not be found in any patient with $Q$ wave myocarditis on the two-dimensional echocardiogram. There was no apparent relation between the region showing $Q$ waves and severity of regional wall motion. However, left ventricular ejection fraction and the number of leads with $Q$ waves showed a significant inverse corrclation $(r=0.629, p=0.038)$. In conrast, four patients with non- $Q$ wave myocarditis showed segmental wall motion abnormality; inferoposterior wall in one and apical segment in three. Three or four weeks later, there were no significant differences in these indices between the two groups, owing mainly to a rapid improvement of myocardial thickening and left ventricular systolic function in the $Q$ wave group.

Five patients with $Q$ wave myocarditis received corticosteroids treatment because their heart failure was highly progressive in spite of usual pharmacological management or intraaortic balloon pump support (2 patients). After corticosteroid treatment for 3 days, left ventricular wall thickening as well as ejection fraction showed a significant improvement (Table II). However, it should be noted that 2 of 5 patients eventually died of repetitive ventricular tachycardia. A case of a 66-year old man for whom corticosteroid treatment could have distinct effects on the clinical course is shown in Figure 3. This patient was admitted to our hospital because of pulmonary edema following upper respiratory infection. Electrocardiogram on admission showed ST elevation and $Q$ waves in $V_{1}-V_{4}$ associated with apparent ST depressions in $V_{5} \cdot V_{6}$. Although left anterior hemiblock might be presumed from limb leads, deep $Q$ waves in chest leads were somewhat unusual as left anterior hemiblock. Left ventricular end-diastolic pressure was elevated ( $18 \mathrm{mmHg}$ ) and cardiogenic shock was noted. Diffuse

Table II. Effects of Corticosteroid Treatment on LV Function

\begin{tabular}{lccc}
\hline & $\begin{array}{c}\text { Before } \\
(\boldsymbol{n}=5 ; 4)\end{array}$ & $\begin{array}{c}\text { After } \\
(\boldsymbol{n}=4)\end{array}$ & $p$ \\
\hline LVEDD (mm) & $47 \pm 16 ; 45 \pm 6$ & $47 \pm 3$ & 0.670 \\
Wall thickness (mm) & $31 \pm 7 ; 31 \pm 7$ & $20 \pm 2$ & 0.035 \\
LVEF $(\%)$ & $35 \pm 7 ; 34 \pm 7$ & $69 \pm 16$ & 0.010 \\
\hline
\end{tabular}

Mortality rate: $40 \%$

LVEDD = left ventricular end-diastolic diameter; $L V E F=$ left ventricular ejection fraction. A comparison is made in four patients who underwent echocardiographic examination before and after treatment. 

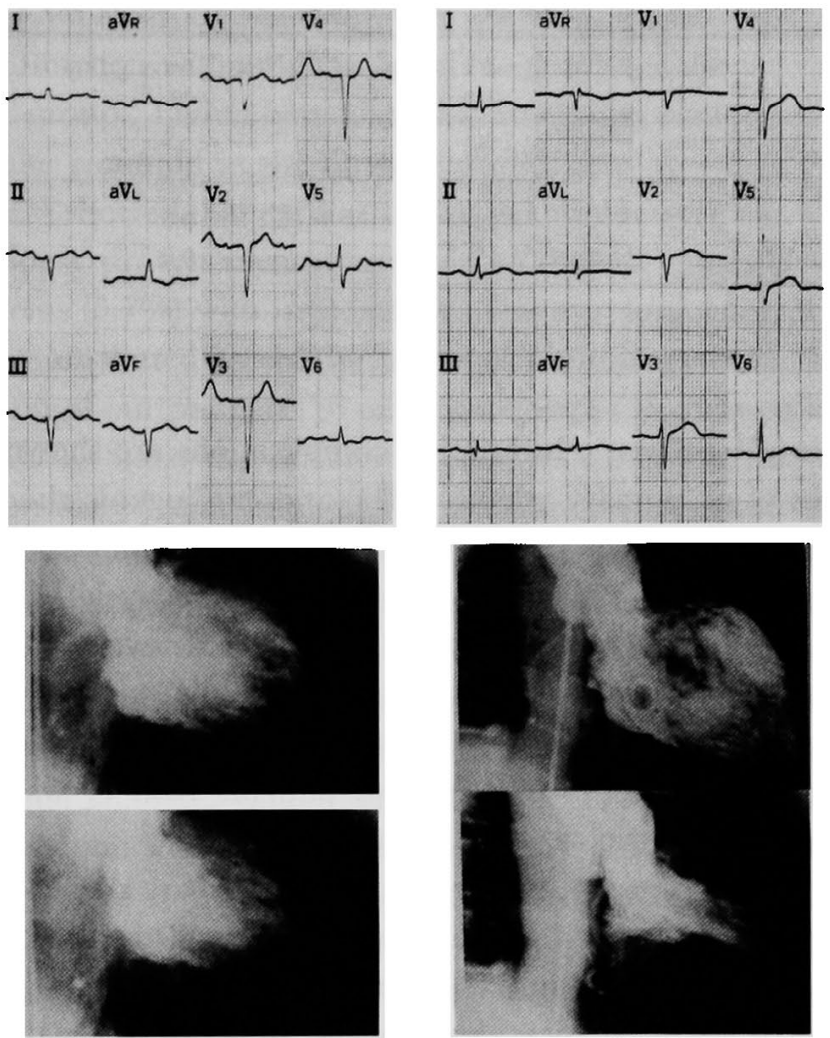

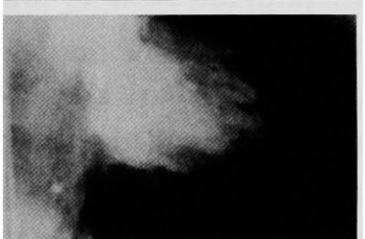

LVEDP $=18 \mathrm{mmHg} ;$ LVEF $=35 \%$

On Admission
LVEDP $=6 \mathrm{mmHg}:$ LVEF $=72 \%$

35 Days Later

Figure 3. Illustrative case (a 66-year old man). Left panel shows electrocardiogram and left ventriculography on admission. Right panel shows those after 35 days. LVEDP = left ventricular end-diastolic pressure.

Table III. Incidence of Severe In-hospital Complications

\begin{tabular}{lccc}
\hline & $\begin{array}{c}\text { Q wave group } \\
(n=11)\end{array}$ & $\begin{array}{c}\text { Non-Q wave group } \\
(n=13)\end{array}$ & $p$ \\
\hline Pulmonary edema & $9(82)$ & $0(0)$ & $<0.001$ \\
Cardiogenic shock & $6(55)$ & $0(0)$ & 0.002 \\
Advanced AV block & $5(46)$ & $1(8)$ & 0.006 \\
Bundle branch block & $6(55)$ & $2(15)$ & 0.043 \\
Ventricular tachycardia/ventricular fibrillation & $6(55)$ & $2(15)$ & 0.043 \\
\hline
\end{tabular}

( ): \%

hypokinetic motion and a decreased ejection fraction of $35 \%$ were found on a left ventriculogram. Thirty five days later, $Q$ waves as well as ST depressions had already disappeared and left ventricular wall motion had also improved to normal contraction. 
Table IV. Follow-up Results

\begin{tabular}{lccr}
\hline & $\begin{array}{c}\text { Q wave group } \\
(n=8)\end{array}$ & $\begin{array}{c}\text { Non-Q wave group } \\
(n=12)\end{array}$ & $p$ \\
\hline Follow-up (month) & $69 \pm 23$ & $45 \pm 25$ & 0.043 \\
Cardiac death & $0(0)$ & $0(0)$ & $>0.999$ \\
Heart failure & $1(13)$ & $0(0)$ & 0.209 \\
NYHA $\geqq$ class III & $1(13)$ & $0(0)$ & 0.209 \\
\hline()$\%$ & & &
\end{tabular}

In-hospital complications: In-hospital complications are shown in Table III. High incidences of pulmonary edema $(82 \%)$ and cardiogenic shock $(55 \%)$ were observed in the $Q$ wave group, while none of these complications were seen in the non-Q wave group ( $p<0.001$ and $p=0.002$, respectively). Conduction disturbances such as bundle branch block or advanced atrioventricular block were also more common in the $Q$ wave group than in the non- $Q$ wave group. In addition, ventricular tachycardia was more common in patients with $Q$ wave myocarditis $(55 \%$ vs $15 \%, p=0.043)$. Two patients with $Q$ wave myocarditis were complicated by ventricular fibrillation.

Follow-up: Follow-up results after hospital discharge are shown in Table IV. A mean follow-up interval after hospital discharge was $69 \pm 23$ months in the $Q$ wave group and $45 \pm 25$ months in the non-Q wave group, respectively $(p=0.043)$. There was no cardiac death in the two groups. One patient with $Q$ wave myocarditis was re-admitted to our hospital because of left side heart failure at 72 months following the first admission. On the echocardiogram, left ventricular end-diastolic diameter increased from $50 \mathrm{~mm}$ to $56 \mathrm{~mm}$ and left ventricular ejection fraction decreased from $49 \%$ to $26 \%$ between the first and second admission.

\section{Discussion}

In acute myocarditis $Q$ waves are often seen, however, they are easily overlooked because of their brief appearance. Previous studies have shown little concern for the differences in serial $\mathrm{CK}$ values as well as echocardiographic findings, in-hospital complications, and long-term prognosis between patients with $Q$ wave myocarditis and non- $Q$ wave myocarditis. This study consisted of 24 patients demonstrated a clinically suspected acute myocarditis and examined these aspects.

Severity of disease: In regard to the severity in the acute stage, this study demonstrates three major findings. First, the $Q$ wave group had a higher incidence of abnormal $\mathrm{CK}$ values as well as higher maximum $\mathrm{CK}$ values compared 
with the non-Q wave group. Second, left ventricular wall thickening and decreased systolic function without chamber dilatation were the important features in the $Q$ wave group. Third, hemodynamic instability and conduction disturbances were quite common in the $Q$ wave group.

Smith et $a l .{ }^{13)}$ showed that cardiac troponin I values were elevated in $35 \%$ of patients with biopsy proven myocarditis, while CK-MB values were elevated in only a few patients $(5.7 \%)$ with myocarditis. This low frequency of elevated CK values might be attributed to the inclusion of chronic patients. In contrast, a higher proportion of our patients ( 16 of 24 patients; 67\%) showed abnormal CK values $\geqq 220 \mathrm{IU} / l$. Since the duration of illness was comparable in the two groups, it is considered that both higher maximum CK values and a higher prevalence of abnormal $C K$ values in the $Q$ wave group is mainly due to greater myocyte injury in patients with $Q$ wave myocarditis than in those with non- $Q$ wave myocarditis.

Echocardiographic findings on admission in $Q$ wave myocarditis were characterized by myocardial thickening, diffuse left ventricular wall motion abnormality and normal chamber dimension, while in non- $Q$ wave myocarditis, left ventricular contraction showed a less diffuse impairment or segmental asynergy. These differences were statistically significant. Previous studies have also shown that left ventricular dysfunction is common $(69 \%)$, particularly in patients with congestive heart failure $(88 \%)$ and in some myocarditis patients with transient myocardial thickening. ${ }^{17,15)} I t$ is possible that the appearance of $Q$ waves is due to profound myocardial injury and consequently causes diffuse left ventricular dysfunction.

Rapid improvement of left ventricular dysfunction and wall thickening was another important feature in the $Q$ wave group. The mechanisms of reversible left ventricular hypertrophy and dysfunction might largely depend on inflammatory changes or tissue edema rather than gross myocardial necrosis. In such a situation, corticosteroid treatment may interfere with the natural course of left ventricular function. Four of our 5 patients who received high doses of methylprednisolone for three days seemed to show a considerable improvement in left ventricular systolic function as well as myocardial thickening. However, two patients died of repetitive ventricular tachycardia and fibrillation. The effects of corticosteroids or immunosupressive therapy in the acute stage of myocarditis remains to be resolved. Experimental studies suggest that corticosteroids or immunosuppressive treatment is deleterious for patients with myocarditis because these treatments enhance viral replication. ${ }^{16,17)}$ However, some clinical studies have shown that both histologic findings and left ventricular function are often improved by immunosuppression. ${ }^{18-20)}$ Recently, the Myocardial Treatment Trial, ${ }^{21)}$ a randomized trial of immunosuppression, concluded that this treatment 
was not beneficial in most patients with histologically confirmed myocarditis. The Multicenter Giant Cell Myocarditis Study Group ${ }^{22}$ has suggestd that immunosuppression may prolong the time to transplantation or death in patients with giant-cell myocarditis. Myocarditis shows a wide clinical spectrum; some cases show an acute onset while others an obscure onset. Our five patients were characterized by prominent myocardial thickening, more depressed left ventricular ejection fraction and progressive heart failure with marked conduction disturbances in spite of the use of inotropic agents or intraaortic balloon pump support. A further randomized study may be needed to evaluate the efficacy of this treatment in these selected patients.

Deteriorated hemodynamics (pulmonary edema or cardiogenic shock) was quite common in the $Q$ wave group. There were no such complications in non$Q$ wave myocarditis. The conduction disturbances were also common in the $Q$ wave group; seven of 11 patients had either advanced atrioventricular block or bundle branch block; five of them had both. In contrast, only two patients in the non- $Q$ wave group had these complications. Previous studies have shown that intraventricular conduction disturbances are closely related to left ventricular dysfunction. ${ }^{6,23)}$ Among our 7 patients with $Q$ wave myocarditis and the conduction disturbances, two patients died during hospitalization, one showed persistent right bundle branch block, and one needed a permanent pacemaker. This indicates that more extensive involvement in working myocardium as well as the conduction system might have occurred in these patients. Patients with $Q$ waves and conduction disturbances may be one of the features of fluminant myocarditis.

Prognosis: Several studies have reported that myocarditis is one of the possible causes of dilated cardiomyopathy. ${ }^{24}{ }^{26}$ However, few clinical data are available in regard to the prognosis of patients with abnormal $Q$ waves. Take et al. ${ }^{1}$ reported in-hospital deaths in 2 out of 10 patients $(20 \%)$ with $Q$ wave myocarditis and 1 out of 6 patients $(17 \%)$ with non- $Q$ wave myocarditis. During the follow-up period, there were no deaths or recurrent heart failure in 8 patients with $Q$ wave myocarditis. In another study, ${ }^{6} 2$ of 3 patients associated with the combination of $Q$ waves and ST elevations showed a rapidly progressive and fatal course. Our study showed that a higher in-hospital mortality rate was noted in $Q$ wave myocarditis $(27 \%)$ compared to non-Q wave myocarditis $(8 \%)$, but this difference was not statistically significant. During the follow-up period, no death was observed in either group. One patient with $Q$ wave myocarditis was re-admitted to our hospital and his clinical presentation was similar to dilated cardiomyopathy. More cases may be necessary to elucidate the long-term prognosis of $Q$ wave myocarditis.

Conclusions: $Q$ wave myocarditis shows more thickened myocardium, de- 
pressed left ventricular function and a higher incidence of severe complications in the acute stage compared to non- $Q$ wave myocarditis. Various therapeutic managements to save deteriorated hemodynamics and/or advanced heart block would be needed urgently in $Q$ wave myocarditis. However, the majority of patients who survive an acute illness show good recovery of left ventricular function. In general, a favorable long-term prognosis may be expected in both types of myocarditis.

\section{References}

1. Nakashima H, Honda Y, Katayama T. Serial electrocardiographic findings in acute myocarditis. Intern Med 1994; 33: 659-66.

2. Miklozek CL, Crumpacker CS, Royal HD, Come PC, Sullivan JL, Abelmann WH. Myocarditis presenting as acute myocardial infarction. Am Heart J 1988; 115: 768-76.

3. Dec GW, Waldman H, Southern J, Fallon JT, Hutter AM, Palacios I. Viral myocarditis mimicking acute myocardial infarction. J Am Coll Cardiol 1992; 20: 85-9.

4. Narula J, Khaw BA, Dec GW, et al. Brief report: recognition of acute myocarditis masquerading as acute myocardial infarction. N Engl J Med 1993; 328: 100-04.

5. Smith WG, Park S. Coxsackie B myopericarditis in adults. Am Heart J 1970; 80: 34-46.

6. Morgera $\mathrm{T}$, Lenarda $\mathrm{AD}$, Dreas $\mathrm{L}$, et al. Electrocardiography of myocarditis revisited: clinical and prognostic significance of electrocardiographic changes. Am Heart J 1992; 124: 455-67.

7. Take M, Sekiguchi $\mathbf{M}$, Hiroe $\mathbf{M}$, Hirosawa $\mathbf{K}$. Long-term follow-up of electrocardiographic findings in patients with acute myocarditis proven by endomyocardial biopsy. Jpn Circ J 1982; 46: 1227-34.

8. Aretz HT, Billingham ME, Edwards WD, et al. Myocarditis: a histologic definition and classification. Am J Cardiovasc Pathol 1986; 1: 3-14.

9. Sahn DJ, De Maria A, Kisslo J, Weyman A. The Committee on M-Mode Standardization of the American Society of Echocardiography: recommendations regarding quantitation in $\mathrm{M}$-mode echocardiography; results of a survey of echocardiographic measurements. 1978; Circulation 58: 107283.

10. Teichholtz LE, Kreulen T, Herman MV, Gorlin R. Problems in echocardiographic volume determinations: echocardiographic-angiographic correlations in the presence or absence of asynergy. Am $\mathrm{J}$ Cardiol 1976; 37: 7-11.

11. Steiner RM, Levin DC. Radiology of the heart. In: Braunwald, editor. Heart Disease. Philadelphia: Saunders Company, 1997: 204-39.

12. Willems JL, Robles De Medina EO, Bernard R, et al. Criteria for intraventricular conduction disturbances and pre-excitation. J Am Coll Cardiol 1985; 5: 1261-75.

13. Smith SC, Ladenson JH, Mason JW, Jaffe AS. Elevations of cardiac troponin I associated with myocarditis: experimental and clinical correlates. Circulation 1997; 95: 163-8.

14. Pinamonti B, Alberti E, Cigalotto A, et al. Echocardiographic findings in myocarditis. Am J Cardiol 1988; 62: 285-91.

15. Hauser AM, Gordon S, Cieszkowski J, Timmis GC. Severe transient left ventricular "hypertrophy" occurring during acute myocarditis. Chest 1983; 83: 275-7.

16. Kilbourne ED, Wilson CB, Perrier D. The induction of gross myocardial lesions by a Coxsackie (Pleurodynia) virus and cortisone. 1956; J Clin Invest 35: 362-70.

17. McManus BM, Chow LH, Wilson JE, et al. Direct viral injury by enterovirus: a central role in the evolution of murine myocarditis. Clin Immunol Immunopathol 1993; 68: 159-69.

18. Salvi $A$, Lenarda $A D$, Dreas $\mathrm{L}$, Silvestri $\mathrm{F}$, Camerini $\mathrm{F}$. Immunosuppressive treatment in myocarditis. Int J Cardiol 1989; 22: 329-38.

19. Hobbs RE, Pelegrin D, Ratliff NB, Silverman CB, Rincon G, Sterba R, Badhwar K. Lymphocytic myocarditis and dilated cardiomyopathy: treatment with immunosuppressive agents. Cleve Clin J Med 
1989; 56: 628-35.

20. Jones SR, Herskowitz A, Hutchins GM, Baughman KL. Effects of immunosuppressive therapy in biopsy-proved myocarditis and borderline myocarditis on left ventricular function. Am J Cardiol 199l; 68: 370-76.

21. Mason JW, O'Connell JB, Herskowitz A, et al. A clinical trial of immunosuppressive therapy for myocarditis. N Engl J Med 1995; 333: 269-75.

22. Cooper LT, Berry GJ, Shabetai R. Idiopathic giant-cell myocarditis: natural history and treatment. N Engl J Med 1997; 336: 1860-6.

23. Matsuura H, Palacios IF, Dec GW, et al. Intraventricular conduction abnormalities in patients with clinically suspected myocarditis are associated with myocardial necrosis. Am Heart J 1994; 127: 12907.

24. Zee-Cheng C-S, Tsai CC, Palmer DC, Codd JE, Pennington DG, Williams GA. High incidence of myocarditis by endomyocardial biopsy in patients with idiopathic congestive cardiomyopathy. J Am Coll Cardiol 1984; 3: 63-70.

25. Dec GW, Palacios IF, Fallon JT, et al. Active myocarditis in the spectrum of acute dilated cardionyopathies: clinical features, histologic correlates, and clinical outcone. N Engl J Med 1985; 312 : "it' 885-90.

26. Kasper EK, Agema WRP, Hutchins GM, Deckers JW, Hare JM, Baughman KL. The causes of dilated cardiomyopathy: a clinicopathologic review of 673 consecutive patients. J Am Coll Cardiol 1994; 23: 586-9. 